\title{
A Secondary Amyloidosis Associated with Systemic Lupus Erythematosus
}

\author{
Tomoko Betsuyaku, Tomoaki Adachi, Hitoshi Haneda, Junichi Suzuki, Masaharu Nishimura, \\ Shosaku ABE*, Tomoo Ito**, Yasunori FujIOKA** and Yoshikazu KawaKami
}

\begin{abstract}
Secondary amyloidosis associated with systemic lupus erythematosus has rarely been reported. A 57-year-old female had been diagnosed as having possible systemic lupus erythematosus, although her clinical course was not typical. About one year after the diagnosis, treatment was begun with prednisolone because of progressive renal dysfunction, thrombocytopenia and low serum levels of complements. Recurrent diarrhea and gastrointestinal bleeding soon developed, then amyloidosis was revealed in the stomach and duodenum. Postmortem examination confirmed systemic amyloidosis. We discuss the significance of this rare association of systemic lupus erythematosus and secondary amyloidosis.
\end{abstract}

(Internal Medicine 32: 391-394, 1993)

Key words: gastrointestinal bleeding, renal amyloidosis, Congo red staining

\section{Introduction}

Secondary amyloidosis is a well-recognized complication of chronic inflammatory, rheumatic and malignant diseases $(1,2)$. However, the association of amyloidosis and systemic lupus erythematosus (SLE) seems very unusual $(3,4)$. In most of the reported cases, the patients had suffered from longstanding SLE $(5,6)$ or another well-known amyloid-associated disorder in addition to $\operatorname{SLE}(7,8)$. In this case, secondary amyloidosis was acutely developed with symptoms of diarrhea and gastrointestinal bleeding in a patient who had been diagnosed as having possible SLE.

\section{Case Report}

A 57-year-old female was referred to the hospital for evaluation of an abnormal chest shadow in October 1989 (Fig.1). On admission she also had slight pain in her left wrist associated with morning stiffness for ten minutes, although she had no respiratory complaints. Three years previously, she had had transient polyarticular arthralgia and been treated with traditional Chinese medicine. There was a history of appendectomy when she was twenty years old. She had had hypertension and been treated with antihypertensive drugs for one year. There was no family history of either connective tissue disease or amyloidosis.
On admission her blood pressure was $164 / 96 \mathrm{mmHg}$, pulse was $78 / \mathrm{min}$ and regular and body temperature was $36.2^{\circ} \mathrm{C}$. On physical examination, a tender swelling of the left wrist and finger joints without deformity was noted. Radiographs of affected joints did not show loss of articular cartilage or bone errosions. A fine crackle was heard at the back in the inspiratory phase. Erythema, photosensitivity, Raynaud's phenomenon and extremity edema were not found.

On laboratory examination impaired renal function was found with a serum creatinine level of $1.2 \mathrm{mg} / \mathrm{dl}, \mathrm{BUN}$ of $57 \mathrm{mg} /$ $\mathrm{dl}$ and a creatinine clearance of $40.0 \mathrm{ml} / \mathrm{min}$. Proteinuria amounted to $1.5 \mathrm{~g} / \mathrm{day}$. In the urinary sediment microhematuria was present. The ESR was $91 \mathrm{~mm}$ in the 1 st hour and C-reactive protein was 1+. False-positive tests for syphilis were not found. The data of autoantibodies on admission is listed in Table 1. Antinuclear antibodies, anti-DNA antibodies and anti-ssDNA were positive. But anti-dsDNA was negative. Serum levels of complement components and $\mathrm{CH}_{50}$ remained almost normal $\left(\mathrm{C}_{3} ; 127 \mathrm{mg} / \mathrm{dl} \mathrm{N}: 46-100, \mathrm{C}_{4} ; 17 \mathrm{mg} / \mathrm{dl} \mathrm{N}: 17-57\right.$ and $\mathrm{CH}_{50}$; $38.7 \mathrm{U} / \mathrm{ml} \mathrm{N}: 30.0-45.0$ ). One of the circulating immune complexes was elevated (anti- $\mathrm{C}_{3} ; 220 \mu \mathrm{g} / \mathrm{ml} \mathrm{N}: 20>$ ). WBC was $6,200 / \mu 1, \mathrm{RBC} 386 \times 10^{4} / \mu 1$ and platelets $10.2 \times 10^{4} / \mu 1$. Electrolytes, FBS, GOT, GPT, LDH, lipids, bilirubin, ECG, respiratory function test and arterial gas analysis were all normal. Occult bleeding in stool was negative by latex fixation test. Total serum protein was $6.8 \mathrm{~g} / \mathrm{dl}$ with albumin $53.2 \%$,

From the First Department of Internal Medicine, School of Medicine, Hokkaido University, Sapporo, *the Third Department of Internal Medicine, Sapporo Medical University, Sapporo (present) and **the Second Department of Pathology, School of Medicine, Hokkaido University, Sapporo

Received for publication July 6, 1992; Accepted for publication March 17, 1993

Reprint requests should be adressed to Dr. Tomoko Betsuyaku, the First Department of Internal Medicine, School of Medicine, Hokkaido University, Kita15, Nishi 7, Kita-ku, Sapporo 060, Japan 


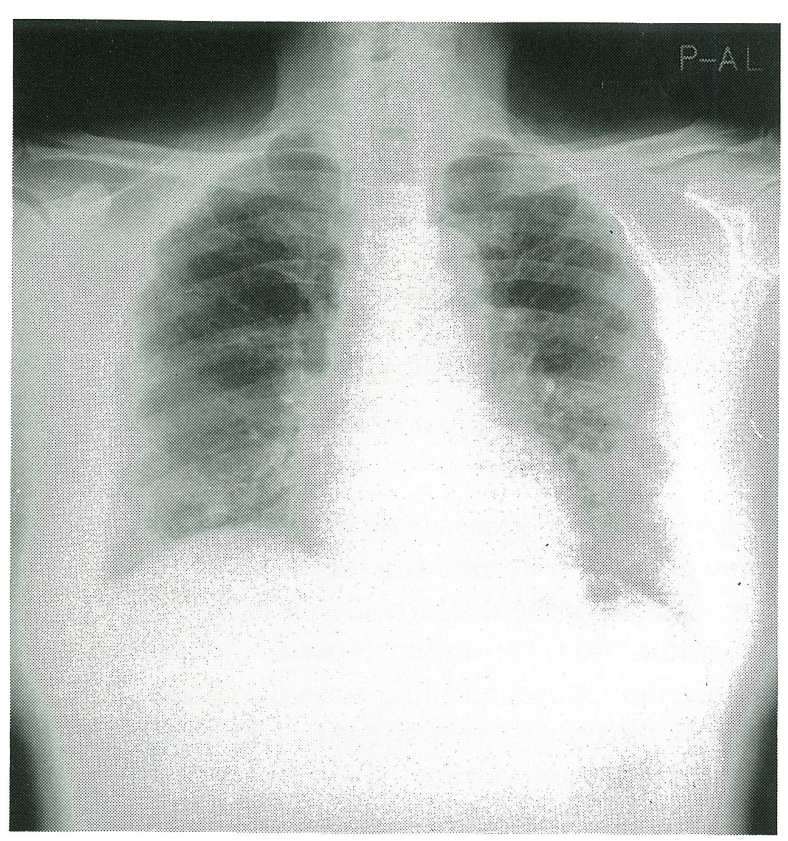

Fig. 1. Chest radiograph on admission, showing reticulonodular shadows in both lower lung fields.

Table 1. Autoantibodies on Admission

\begin{tabular}{lc}
$\begin{array}{l}\text { Antinuclear antibodies } \\
\text { (speckled pattern) }\end{array}$ & $20 \times$ \\
Anti-DNA Ab & $27 \mathrm{U} / \mathrm{ml}$ \\
LE test & $(-)$ \\
RA test & $(+)$ \\
Anti-RNA Ab & $(-)$ \\
Anti-Sm Ab & $(-)$ \\
SS-A Ab & $(-)$ \\
SS-B Ab & $(-)$ \\
Anti-Scl-70 Ab & $(-)$ \\
Anti-ssDNA Ab & $28 \mathrm{U} / \mathrm{ml}$ \\
Anti-dsDNA Ab & $(-)$ \\
& \\
\hline
\end{tabular}

gammaglobulin 22.7\%. IgA and IgM were normal, but IgG was slightly elevated $(1,752 \mathrm{mg} / \mathrm{dl})$.

A chest radiograph showed reticulonodular shadows in both lower lung fields (Fig.1). Bronchoalveolar lavage fluid revealed a remarkable increase of lymphocytes $(55.9 \%)$, and the CD4/8 ratio was elevated to 4.75 . A transbronchial lung biopsy specimen revealed infiltration of lymphocytes and fibrosis in the septum. A diagnosis of interstitial pneumonia in association with connective tissue disease was established. A renal biopsy, performed to evaluate the renal dysfunction, revealed that almost all glomeruli showed hyalinosis due to sclerosis. In the remaining glomeruli, mesangial cell proliferation and some thickening of the basement membrane were found (Fig. 2).

On immunofluorescence examination, depositions of IgM, $\mathrm{C}_{1 \mathrm{q}}$ and $\mathrm{C}_{3}$ were noted along the basement membrane and in the mesangium. These histopathological findings were defined as lupus nephritis, and classified as category VI, advanced sclerosing glomerulonephritis. Amyloid deposits were not de-

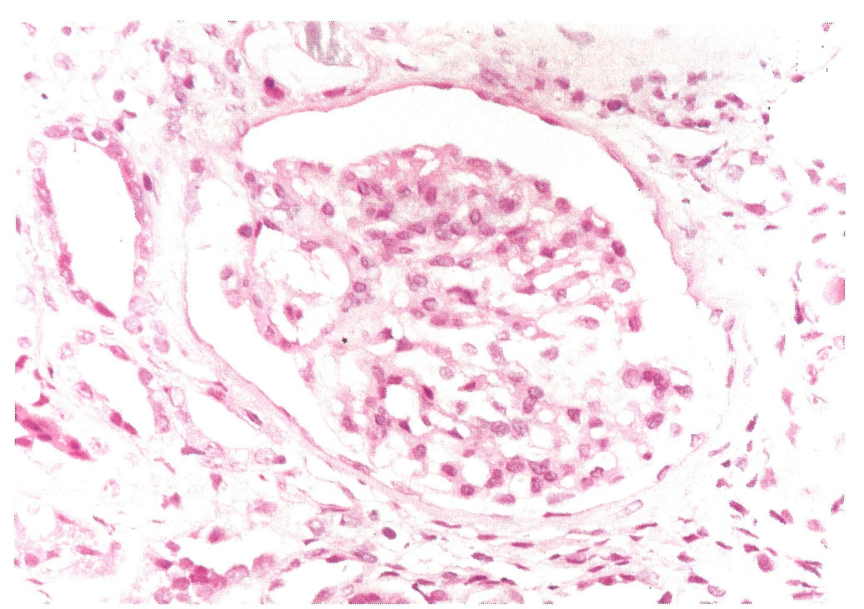

Fig. 2. A renal biopsy specimen showing one of a few remaining glomeruli which has slight mesangial cell proliferation and thickening of the basement membrane (HE staining, $\times 540$ )

tected at that time. A diagnosis of SLE was highly suspected, based on the presence of arthritis, proteinuria, thrombocytopenia, and positive tests for antinuclear antibodies and anti-DNA, consisting with interstitial pneumonia and lupus nephritis.

She was discharged in fair clinical condition without any treatment such as steroids in February 1990. Eleven months later she was readmitted because of bronchopneumonia, which was improved by administration of antibiotics. At that time, the ESR was $80 \mathrm{~mm}$ in the 1 st hour, $\mathrm{RBC}$ was $370 \times 10^{4} / \mu 1$, $\mathrm{Hb}$ was $10.4 \mathrm{~g} / \mathrm{dl}$. Antinuclear antibodies were positive with a titre of 1:160 with homogenous and speckled pattern and anti-DNA antibodies were negative. $\mathrm{CH}_{50}$ was $33.3 \mathrm{U} / \mathrm{ml}$. Since diarrhea developed during the subsequent weeks, upper gastrointestinal endoscopy and colon endoscopy were performed; these showed no abnormalities except for some linear erosions in the antrum of the stomach. In February 1991 treatment with $60 \mathrm{mg}$ /day of prednisolone was started because of progression of renal dysfunction (BUN; $57 \mathrm{mg} / \mathrm{dl}, \mathrm{Cr} ; 1.8 \mathrm{mg} / \mathrm{dl}$, Ccr; $24.6 \mathrm{ml} / \mathrm{min}$, total urinary protein; $1-1.5 \mathrm{~g} /$ day) and thrombocytopenia $\left(1.0 \times 10^{4} / \mathrm{ml}\right)$, lymphocytopenia $(756 / \mathrm{ml})$ and reduced serum levels of complement components $\left(\mathrm{C}_{3} ; 35 \mathrm{mg} / \mathrm{dl}, \mathrm{C}_{4} ; 13 \mathrm{mg} / \mathrm{dl}\right)$, which were thought to be an exacerbation of SLE. The treatment with prednisolone did not result in improvement of these clinical features. Abdominal pain and melena developed at the beginning of April without abnormalities in clotting tests. Upper gastrointestinal endoscopy showed diffuse reddish erosion and small excaved lesions in the antrum of the stomach and yellowish-white granular lesions in the descending part of duodenum (Figs. 3 and 4). Biopsy specimens revealed massive amorphous deposition only in the submucosal vessels, which led to vessel wall thickening. This was confirmed to be amyloid deposition by Congo red staining (Fig. 5). The gastric biopsy specimen taken three months previously was re-examined for the presence of amyloid, but no deposition was detected. Blood transfusion was repeated and totally amounted to $3,000 \mathrm{ml}$ against anemia caused by recurrent bleeding. However, her 


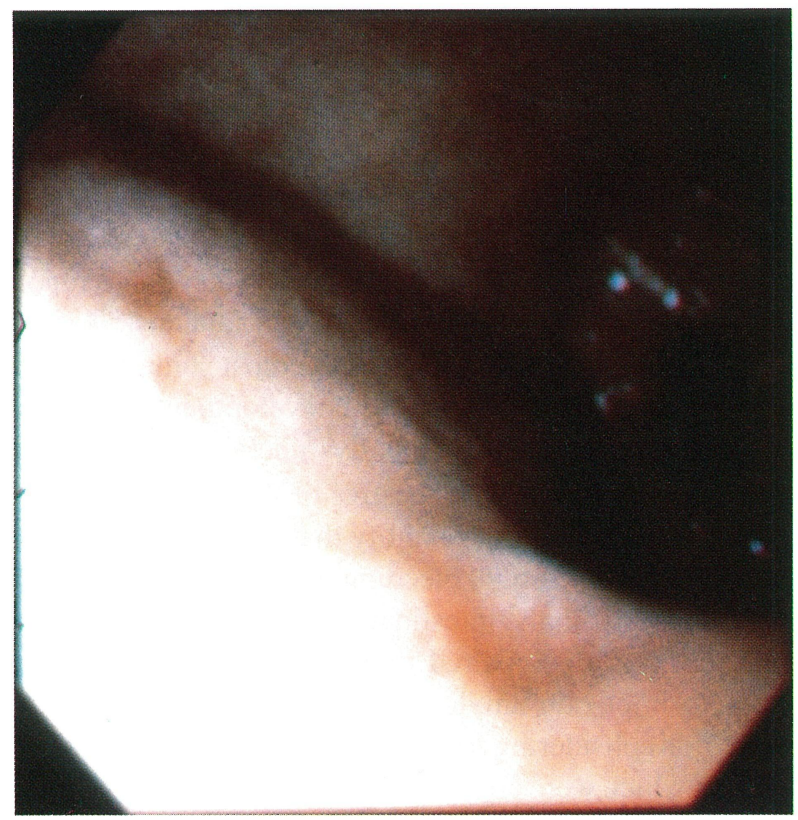

Fig. 3. Upper gastrointestinal endoscopy, demonstrating diffuse reddish erosion and small excaved lesions in the antrum.

general condition rapidly deteriorated and she died of sepsis with fungi at the end of April.

At autopsy the diagnosis of systemic amyloidosis was confirmed with the deposition of amyloid in the total gastrointestinal tract, kidneys, liver, heart, spleen, skin and other connective tissues. These amyloid deposits lost their stainability with Congo red after treatment with potassium permanganate, which suggested that the amyloid was of the secondary (AA) type. In

Fig. 5. A duodenal specimen showing obvious amyloid deposition of the vascular wall in the lamina propria mucosae. (Congo red staining, $\times 270$ )

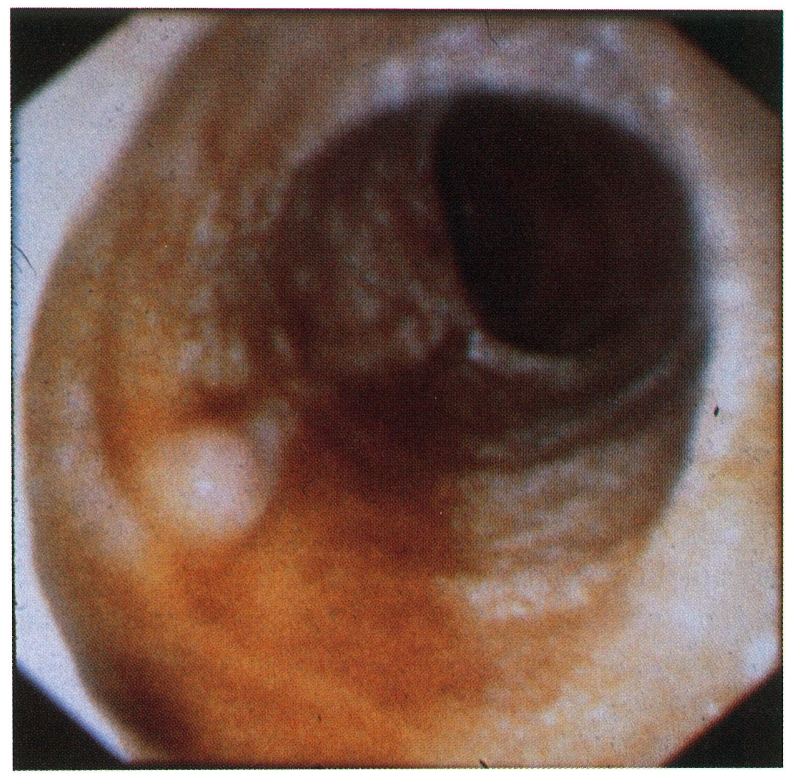

Fig. 4. Endoscopic finding of duodenum, showing yellowish-white granular lesions distributed throughout the descending part.

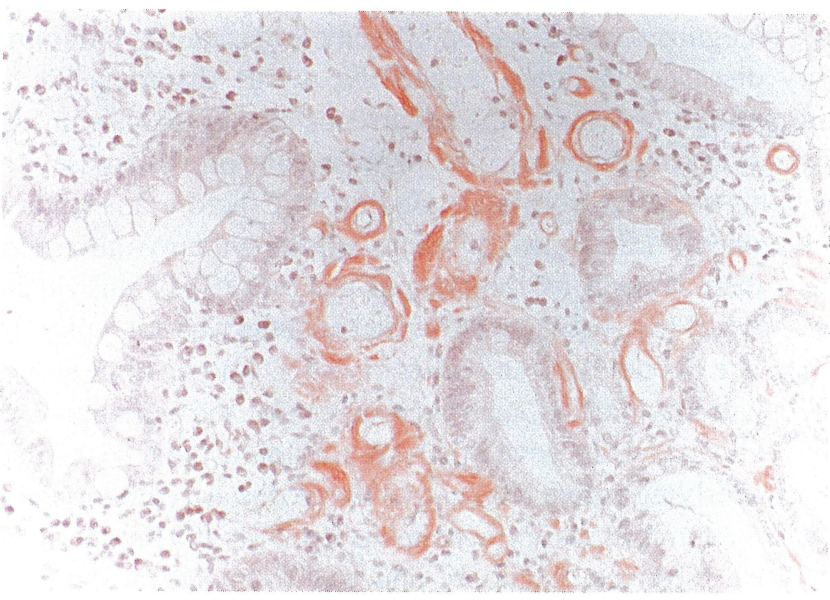

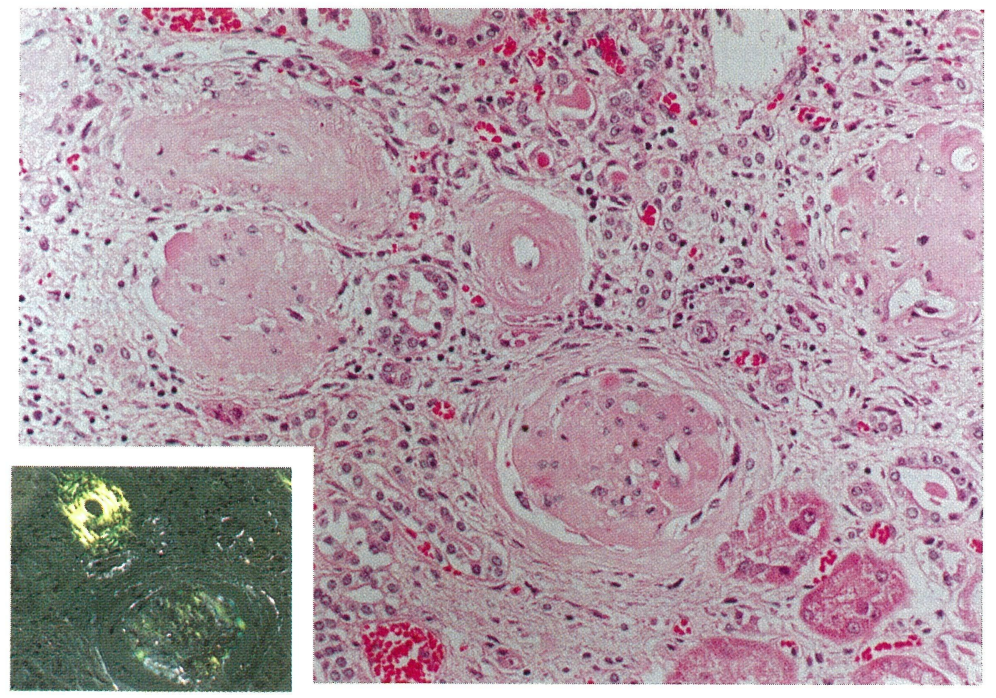

Fig. 6. Renal tissue obtained by autopsy in April 1991. Homogenous eosinophilic material of the vascular wall and glomeruli are present diffusely. (HE stain, $\times 270$ ). $\times 185)$.

[Insert] Birefringence of amyloid deposit (Congo red staining and polarized light, 
the kidney specimens massive amyloid deposits were found in vascular walls and glomeruli (Fig. 6). Regarding the lung, pulmonary fibrosis was revealed, showing honeycomb lung in the bilateral lower lobes.

\section{Discussion}

This patient fulfilled the 1982 revised American Rheumatism Association criteria for the classification of SLE (9), since she had symptoms of more than four of the necessary diagnostic criteria (arthritis without bone destruction, profuse proteinuria, lymphocytopenia and thrombocytopenia, positive anti-DNA antibodies and positive antinuclear antibodies). Histopathological findings of her renal biopsy specimen were consistent with lupus nephritis. Concerning her interstitial pneumonia, elevation of $\mathrm{CD} 4 / 8$ ratio is not consistent with some previous reports demonstrating decreased $\mathrm{CD} 4 / 8$ ratio in SLE, although its diagnostic value is still controversial $(10,11)$. The reasons for the high CD4 /8 in the present case may be explained by the atypical interstitial pneumonia leading to fibrosis for SLE and the patient's old age. Secondary amyloidosis is a recognized complication of many longstanding inflammatory diseases, including connective tissue disorders $(1,2)$. Surprisingly, however, the occurrence of amyloidosis in SLE seems very uncommon $(3,4)$. In most of the previous cases, the patients suffered from longstanding SLE before amyloidosis developed $(5,6)$, or had another well-known amyloid-associated disease such as rheumatoid arthritis (7) or ankylosing spondylitis (8). Although her clinical course was not typical for SLE, she did not have any other disorders known to be associated with amyloidosis, such as chronic infection or malignancy. We believe that she developed AA amyloidosis as the result of her underlying active connective tissue disease categorized in SLE. Systemic lupus erythematosus itself rapidly progressed in the year after the diagnosis, and was followed by the acute occurrence of amyloidosis soon after steroid treatment was begun.

Proposed mechanisms of amyloid deposits in the course of SLE include the potential role of other autoimmune diseases, long-term effects of steroid treatment, and chance alone (4). Although the particular reason is unknown in this case, it seems hard to ascribe the secondary amyloidosis to the treatment with prednisolone, because it was given only for a short time. Acute exacerbation of SLE in three months may have had some effects on the development of amyloidosis, or amyloidosis may have made her condition unresponsive to the steroid treatment.

In this case the careful examination of gastrointestinal bleeding led to the ante-mortem diagnosis of secondary amyloidosis. Gastrointestinal involvement of amyloidosis is well recognized and is demonstrated in almost all patients with systemic amyloidosis (12). However, early detection is very rare because it is sometimes asymptomatic, and symptoms such as nausea, vomiting, abdominal pain, diarrhea or gastrointestinal bleeding are not pathognomonic. Bleeding problems are reported to be common, although usually mild, occurring in $41 \%$ of amyloidosis cases, of which $18 \%$ included gastrointestinal tract bleeding (13). Our patient had repeated episodes of melena in the absence of any abnormalities in clotting tests. This suggested that the bleeding was caused by amyloid deposition in blood vessels rather than by coagulation abnormalities. Vascular fragility and obliteration of the lumen caused by amyloid deposition are thought to lead to bleeding. It is reported that significant gastrointestinal bleeding is fatal in $45 \%$ of such cases (14), although renal failure is the most common cause of death (15). In this case, the sudden development of melena in addition to the progressive renal dysfunction were the factors leading to a clinical clue suggestive of the superimposition of amyloidosis. There is a possibility that her inveterate diarrhea preceding gastrointestinal bleeding was the initial symptom of amyloid deposits in the intestinal walls and autonomic nerves, which resulted in impaired motor activities. However, it is difficult to implicate amyloidosis as the only cause of renal dysfunction considering concomitant global sclerosis and lupus nephritis, which were confirmed in the renal biopsy specimens. Thus, our experience suggests that, although rare, amyloidosis should be a diagnostic consideration when patients with SLE have recurrent undiagnosed diarrhea or gastrointestinal bleeding.

\section{References}

1) Kyle RA, Bayrd ED. Amyloidosis: review of 236 cases. Medicine 54: 271,1975

2) Glenner GG, Page DL. Amyloid, amyloidosis, and amyloidogenesis. Int Rev Exp Pathol 15: 1,1976.

3) Huston DP, McAdam KPWJ, Balow JE, et al. Amyloidosis in systemic lupus erythematosus. Am J Med 70: 320,1981.

4) Carstens PHB, Ogden LL Jr, Peak WP. Renal amyloidosis associated with systemic lupus erythematosus. Am J Clin Pathol 74: 835,1980.

5) ter Borg EJ, Janssen S, van Rijswijk MH, et al. AA amyloidosis associated with systemic lupus erythematosus. Rheumatol Int 8: 141,1988.

6) Pettersson $\mathrm{T}$, Toernroth $\mathrm{T}$, Toetterman $\mathrm{KJ}$, et al. AA Amyloidosis in systemic lupus erythematosus. J Rheumatol 14: 835,1987.

7) Wegelius O. Amyloidosis of the kidneys, adrenals and spleen as a complication of acute disseminated lupus erythematosus treated with ACTH and cortisone. Acta Med Scand 156: 91,1956.

8) Webb S, Segura F, Cervantes F, et al. Systemic lupus erythematosus and amyloidosis. Arthritis Rheum 22: 554,1979.

9) Tan EM, Cohen AS, Fries JF, et al. The 1982 revised criteria for the classification of systemic lupus erythematosus. Arthritis Rheum 25: 1271,1982 .

10) Izumi T, Nagai S, Kitaichi M, et al. Clinical value of the management of BALF cell subsets in interstitial lung disease (abstract). Chest 89: 137S, 1986.

11) Klech $\mathrm{H}$, Hutter C. Clinical guidelines and indications for bronchoalveolar lavage (BAL): Report of the European Society of Pneumology Task Group on BAL. Eur Respir J 3: 937, 1990.

12) Yamada M, Hatakeyama $S$, Tsukagoshi H. Gastrointestinal amyloid deposition in AL (primary or myeloma-associated) and AA (secondary) amyloidosis: Diagnostic value of gastric biopsy. Hum Pathol 16: $1206,1985$.

13) Yood RA, Skinner M, Rubinow A, et al. Bleeding manifestation in 100 patients with amyloidosis. JAMA 249: 1322,1983.

14) Levy DJ, Franklin GO, Rosenthal WS. Gastrointestinal bleeding and amyloidosis. Am J Gastroenterol 77: 422,1982.

15) Browning MJ, Banks RA, Tribe CR, et al. Ten years' experience of an amyloid clinic - A clinicopathological study. Q J Med 54: 213,1984. 